\title{
Prototyping of an Individualized Multi-Material Wrist Orthosis Using Fused Deposition Modelling
}

\author{
Filip Górski1 ${ }^{*}$, Wiesław Kuczko' ${ }^{1}$ Weronika Weiss ${ }^{1}$, Radosław Wichniarek' \\ Magdalena Żukowska' \\ 1 Poznan University of Technology, Chair of Production Engineering and Management, ul. Piotrowo 3, 61-138 \\ Poznan, Poland \\ * Corresponding author's e-mail: filip.gorski@put.poznan.pl
}

\begin{abstract}
The paper presents the design and manufacturing process of an individualized wrist orthosis. The patient's upper limb was 3D scanned and the orthosis was designed using a CAD system. Each part of the orthosis consists of two different materials that fulfill different functions. By using the double-head Fused Deposition Modelling machine it was possible to produce these parts in a single process without the need for additional assembly operations. The orthosis has been tested for mutual fit of parts, strength and comfort of use.
\end{abstract}

Keywords: wrist orthosis, 3D printing, fused deposition modelling, multi-material, FDM, ABS, TPU.

\section{INTRODUCTION}

The orthoses are medical supplies, used for keeping a selected part of patient's body rigid and safe during healing or convalescence. Usually, it is realized by immobilizing and protecting the body around a selected joint from deformations and physical damage. The orthoses may be also used for enforcement of a specific position and mutual orientation of various body parts (e.g. in the case of muscular palsy) [5]. The orthoses available for patients can be divided into two groups by their fitting method. The first group contains the mass produced orthoses, in several sizes (series of types). They are cheaper and widely available, but due to the lack of proper adjustment to the user's body, they often do not work correctly, especially when used over a longer period of time. This criterion is fulfilled in the case of the orthoses from the second group, manufactured for a specific user [2]. A standardized process of production of these orthoses may require the creation of a gypsum positive model of a limb, on the basis of a previous casting or imprint. This is a prolonged process and requires the technician to have certain manual skills, in order to introduce appropriate corrections into a base model. The final product is manufactured by laminating or thermoforming (manually or with aid of vacuum device) on a positive pattern acquired in the previous stage. Then, machining and assembly is required. For better fitting, additional geometry improvements are made directly with involvement of a given patient $[19,24]$. In the case of the wrist hand orthoses (WHOs), it is possible to use sheets of thermoplastic material, shaped directly on the patient's body. After compressing and gluing sheets, a pause is made until the material hardens, then, the created seam is ripped apart. The surplus of the material is removed and assembling elements are added. This method is quicker, but it cannot be used universally for all types of orthoses and also requires manual skills [22, 28]. In modern industry, an effective mass customization can be acquired by changing product design and process control [26], but because of its nature, no method of production of individualized orthoses mentioned above is suitable for the mass, automated production. 
Additive layered manufacturing allows obtaining medical devices with the shapes tailored to the anatomy of a particular patient [11]. In the literature, there are examples of the research related to the design and manufacturing of various types of upper limb orthoses, which, owing to a better fit to patient's body, increase the quality of the healing process $[4,5,21]$. What is more, such orthoses have a lower mass than their traditionally manufactured counterparts, are hygienic and provide good ventilation, which is important regarding the comfort of use [21]. The most commonly used methods of additive manufacturing of orthoses are SLS (Selective Laser Sintering) and FDM (Fused Deposition Modeling), although examples can also be found using SLA (Stereolitography) and MJ (Material Jetting) [11, $22,25]$. In the literature, there are also examples pertaining to the use of the powder-based methods (3DP) to create positive forms, which are then used in the traditional method of manufacturing of orthopaedic supplies [8].

There are commercially available wrist hand orthoses, distinguished by a unique shape or assembly method $[27,29]$. These examples, in addition to usability, clearly focus on the form of the product, attempting to maximize its visual appeal. The used patterns are most often truss-like structures, mimicking the appearance of nature, for example structure of coral, or are the implementation of the Voronoi pattern. Currently, the design of such an orthosis requires experience in the use of 3D graphics modeling software (CAD $3 \mathrm{D})$. As the authors of the paper [17] note, the lack of dedicated, easy-to-use design software significantly limits the possibility of widespread use of the additively manufactured orthoses. They offered their own technical solution, which is so simple and intuitive that it can also be used by the medical personnel with no experience, with traditional CAD 3D systems for engineers. The authors of the work [4] suggest that the software for the design of orthoses should use only the simplest modeling operations, performed directly on the meshes coming from the scanning process.

Regardless of the tool for designing a digital orthosis model, it is necessary to know the anatomy of the patient. It can be obtained by means of standard medical imaging or by measuring the patient's body using the three-dimensional noncontact scanning, for example using the structured light. The latter technique is mainly known for its industrial applications in mechanical engineer- ing; however, its suitability for medical purposes has also been confirmed in many studies [6].

One of the factors limiting the possibility of use of the FDM method in the production of ready-for-use products is the limited range of the available materials or significant differences in the properties with respect to the same material processed by traditional techniques, e.g. injection molding. In the limited scope end parts properties could be improved by FDM process parameters optimization [12]. Additionally, new possibilities of product design are expected in the case of the availability of the methods for manufacturing of multi-material elements, especially with built-in electronic circuits [23]. Currently, it is possible to produce multifunctional products in a single manufacturing process. In such products, certain parts can be made of a rigid material (e.g. PLA), others of a flexible one (e.g. TPU), with others having an ability to conduct electricity $[13,18]$. Multi-material manufacturing can be also understood as the production of a multicolor product, which consists of a single thermoplastic material, enriched with various pigments, determining its variable color after extrusion. It is also possible to discuss the multi-material production in the context of processes, where the support material is different than the building material (e.g. due to the need to wash it out of hard to reach places). In this case, the final product is composed of only one type of material.

In the FDM devices, multi-material manufacturing in scope of a single process can be implemented in several different ways. A purely software solution is the proper control of the NC code, which will stop machine operation at the right moment and will require the manual change of material by the operator. Due to a very small degree of automation and the time-consuming nature of such an operation, it is used in industrial practice only as a last resort, mainly for simple products, where there are only several changes of material throughout the production process, and the transition material, resulting from the extrusion of a mixture of two materials for a certain time, can be concealed inside the product. Another method is the use of several different material feeders in the FDM machine, supplying material to the common nozzle [16]. During the manufacturing process, it is possible to automatically feed and discharge a particular material into the extrusion nozzle. The disadvantage of this solution is the longer extrusion time associated with the 
change of material in the nozzle and the increased material consumption, which is extruded on the additional structure, in order to expel the transition material out of the manufactured component. Moreover, this way of feeding the extrusion nozzle, realized with a bowden type extruder, is not suitable for extruding elastic materials, such as TPU. The third solution involves the FDM devices with multiple extrusion nozzles, fed with various materials [15]. It is a solution that fully automates the multiple material part production in a single process, at the same time it does not significantly increase the time needed for its implementation and does not increase the material consumption. On the other hand, it is a more expensive solution, as it requires providing precise positioning of the nozzle during the material change and the lack of physical interaction of the inactive nozzle with the manufactured element. the uncontrolled leakage of material from the inactive nozzle and its deposition on the manufactured component are also frequent problems. This phenomenon should be limited by using lower extrusion temperatures and retraction of the material in the inactive nozzle. In commercial solutions, it is common to find double-headed FDM machines that have nozzles linked with a common kinematic system. There are also technical solutions with a larger number of nozzles and independent kinematic systems. The research works aimed at improving existing machine designs are found as well $[1,10]$.

In [18], the quality of diffusion between various thermoplastic materials (PLA, PET, TPU) in a single FDM process was examined. It was shown that there is a significant reduction in strength properties at the boundary of joining materials, which leads to a weakening of the whole structure. A particularly large reduction in tensile strength was observed when combining PLA and TPU. It indicates an extremely poor tendency to form stable bonds between these thermoplastics. As the authors suggest, the appropriate design of their division surfaces is an important issue in the manufacturing of a product made of multiple materials.

In [7] a hand prosthesis, which was prepared for additive manufacturing using several materials already at the design stage, is presented. Following the e-NABLE project, the authors of the work used, among others, the combination of rigid PLA material and elastic TPU material, proving the suitability of such a solution at the stage of direct testing with the patient.
The authors of the work [24] applied a combination of manufacturing processes using the SLM and SL methods to create a functional arm orthosis. The developed method allows production of a bimaterial product. The core of the product, designed to carry loads, can be made of metal or ceramic alloys, while the outer coating, which is in contact with the skin of the patient, can be manufactured of polymer.

Another approach to the multi-material orthoses was used by the authors of the work [20]. On the low-cost FDM machine, they created elements of an ankle orthosis using a single material - PLA. Then, the areas most exposed to damage due to the load were strengthened by applying a composite layer consisting of carbon fibers and an epoxy resin.

The ABS thermoplast- Poly (Acrylonitrilebutadiene styrene) - is a material used on a huge scale in many industries. It is characterized by good resistance to dynamic loads, dimensional stability and is suitable for processing with various additive manufacturing methods, including FDM. ABS is rigid and resistant to external factors, so it is suitable for manufacturing medical devices, including the mid-surgery supplies that require sterilization $[3,6]$. Although it is allowed to stay in contact with the human body, in the case of the products that will be in contact with the skin, it can be covered with an additional transitional layer, e.g. polyurethane foams or natural, breathable fabrics in order to provide better comfort of use [21].

TPU is a thermoplastic polyurethane elastomer based on polyesters or polyethers. They are characterized by considerable elasticity, as well as very good resistance to dynamic loads and abrasion. Additionally, they are resistant to weather conditions and do not deteriorate in contact with oils and fats. The products made of this material can be sterilized by plasma [14]. In medicine, the biocompatible versions of the TPU material are used in conventional manufacturing of prostheses, implants, support structures for tissue regeneration and artificial blood vessels [9]. The mechanical properties of TPU, such as viscosity and hardness, can be applied in the layered additive manufacturing process of FDM. Moreover, during such processing, no harmful substances are released from TPU [14].

The literature analysis shows that it is possible and reasonable to carry out research on the possibilities of creating multi-material wrist 
orthoses. By combining the properties of the ABS and TPU material, the authors of this paper expect to create an orthosis that provides great comfort of use and minimizes the amount of work required to produce it.

\section{MATERIALS AND METHODS}

The aim of this work was to develop and study a prototype design of a multi-material wrist hand orthosis that could be produced as part of a single manufacturing process, without the need for tedious post processing operations. The conducted work can be divided into the following stages:

- three-dimensional structured light scanning of the upper limb of the user

- processing a set of separate scans into a single triangular mesh representing the anatomical shape of the user's forearm

- modeling of the digital geometry of the orthosis, on the basis of the created triangular mesh

- manufacturing orthosis elements on a selected FDM machine

- tests of orthosis by the user.

The research was not aimed at developing an orthosis structure that would have better healing or protective properties. The basic criterion for the assessment was the provision of comfort of use and the possibility of quick and easy manufacturing of the orthosis on a selected FDM machine. Therefore, a well-known design of the wrist orthosis, used in the convalescence period after breaking the wrist bone, was used as the basis for the design requirements. As an orthosis user, a 23-year old woman was selected.

The main aim of the first stage of research was to obtain the information about the shape of the upper limb of the user. Due to the type of orthosis, the most important part was the part of the metacarpal and the forearm. For this purpose, a low-cost David SLS-3 structured light scanner was used. The measurement was performed on the unclothed right upper limb, which was in the corrected, unforced position. The limb did not change its position during scanning and was permanently supported in two places - in the metacarpal region and in the back of the forearm. The choice of the support areas was to ensure the stability of the limb position during the measurement and to minimally affect the deformation of soft tissues in places that are important for the further process of orthosis design. In total, 8 scans

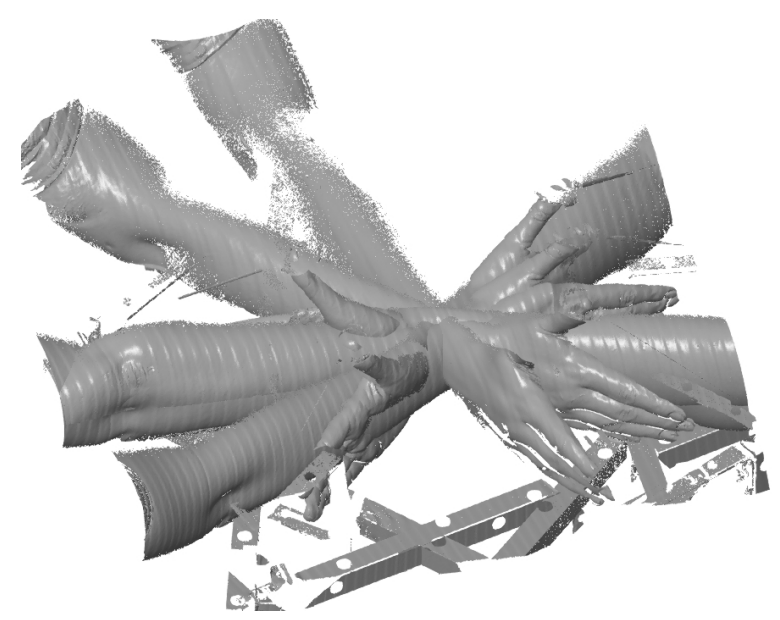

Fig. 1. The raw set of scans, elements of the background and structure supporting the limb visible apart from the user's limb

were taken, each from a different perspective, after the scanner was moved. Figure 1 shows the set of the first 6 raw scans. The data from all the scans were processed in the David 3D Scanning software (used for 3D scanning). Among others, the scanned background elements and the structure supporting the limb were removed. Then the scans were exported to the STL format.

In the next stage of the work, the GOM Inspect software version 7 was used, in which all the scans were reoriented, to obtain a coherent triangular network representing the external structure of the user's limb. In this process, modifications were also introduced to the particular meshes, consisting, among other things, in the removal of excess point data, as well as homogenization and smoothing of areas with high roughness. Figure 2 shows the effect of combining the data from all the 8 scans before cutting them and closing the holes, so the entire surface of the hand was recognized directly by 3D scanning. Additionally, the triangle density in the final mesh was reduced in

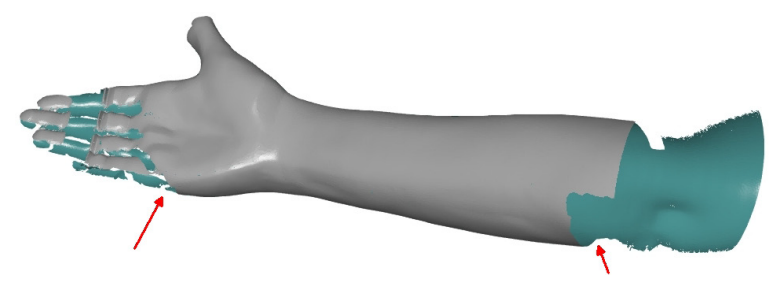

Fig. 2. The triangular mesh created by assembling 8 separate scans of the upper limb of the user. Red arrows indicate limb support places during scanning 
order to facilitate further processing. A reduction coefficient was used to maintain dimensional accuracy, of a deviation not less than $0.4 \mathrm{~mm}$ from the non-reduced mesh.

Creation of a digital model of the orthosis was carried out in the Autodesk Inventor software. With the Mesh Enabler plugin, the triangle mesh obtained in the previous step was converted to a 3D solid. The model was cut to the final dimensions of the orthosis, and then using the thickening / offset option with a value of $1 \mathrm{~mm}$, the surface of the inner side of the orthosis was obtained. The clearance left between the limb and the orthosis was intended to allow compensation for the possible dimensional changes of user's limb, e.g. as a result of swelling. Another offset, made on the basis of the previous one, by a value of $1.5 \mathrm{~mm}$, allowed determining the closing surface for the layer, which was supposed to be elastic and pleasant in contact with the skin. The last offset of the surface was made in just as the previous one, but with a value of $3.5 \mathrm{~mm}$. The obtained surface was the outer surface of the orthosis and was intended to be responsible for stiffening the structure and protecting the limb from external forces. The standard wrist orthoses are made of a thermoplastic material having a thickness of 2-3.5 mm [21]. All the surfaces obtained as a result of consecutive design operations were converted into two solids, separate, but in contact with each. In order to enable the assembly of the orthosis on the patient's body, each block had to be divided into two parts, close in size, along the largest overall dimension. It also enforced the design of assembly catches and gouging along the edge of the division. Its task is to facilitate the installation and removal of the orthosis.

In order to provide better ventilation properties and reduce stresses in the orthosis, the diamondshaped holes were made in the model. The shape, size and arrangement were also selected to maintain adequate stiffness of the entire structure, as well as the possibility of its manufacturing using the FDM method with minimal consumption of the support material. Each of the four solids of the complete design of the orthosis was exported to an STL file, maintaining the common coordinate system.

An FDM machine Raise3D Pro2 Plus was used for the physical fabrication of the orthosis. It has two tippable extrusion heads, ensuring lower rate of material dripping from the inactive nozzle during the layer deposition process. At the same time, the working chamber is large enough for the orthosis to fit completely, oriented verti-
Table 1. Values of the most important parameters of the FDM process

\begin{tabular}{|l|c|}
\hline \multicolumn{1}{|c|}{ Parameter name } & Value \\
\hline $\begin{array}{l}\text { ABS material extrusion } \\
\text { temperature }\end{array}$ & $230^{\circ} \mathrm{C}$ \\
\hline $\begin{array}{l}\text { TPU material extrusion } \\
\text { temperature }\end{array}$ & $220^{\circ} \mathrm{C}$ \\
\hline Table temperature & $100^{\circ} \mathrm{C}$ \\
\hline Basic manufacturing velocity & $40 \mathrm{~mm} / \mathrm{s}$ \\
\hline Layer thickness & $0.25 \mathrm{~mm}$ \\
\hline Number of shells & 2 \\
\hline Full closing/opening layers & $4 / 4$ \\
\hline Infill rate & $40 \%$ \\
\hline Infill pattern & fast honeycomb \\
\hline
\end{tabular}

cally (largest dimension in the $\mathrm{Z}$ axis). Both parts of the orthosis, each consisting of two layers (elastic and rigid material), could be produced as part of a single process. Due to the expected long production time, it was decided to manufacture them separately, so the entire process could be controlled all the time.

As a material for the outer layer of orthosis, black ABS was used, while red TPU was used for the inner layer. In the $\mathrm{NC}$ code preparation program for the FDM machine, the automatic generation of supports was turned off. However, due to the construction of the thumb hole, it was necessary to manually add supports there. They were extruded out of the ABS material. In order to increase the contact surface of the orthosis with the work table, a raft with a $5 \mathrm{~mm}$ offset from the edge of the model was used. Other values of the most important parameters of the manufacturing process are presented in Table 1 . They were selected based on the experience of authors and literature data.

After removing each part of the orthosis from the FDM machine, only a minimal post processing was required, consisting in separation of the support and raft from the product. Then, the orthosis was given to the user for testing.

\section{DISCUSSION}

The low-cost scanner using structured light enabled a quick and efficient gathering of user's anatomical data. Similarly to other research works described in the literature, the greatest deviations and irregularities in the construction of the mesh representing the user's limb were observed near 
Table 2. Production time and material consumption in the FDM process

\begin{tabular}{|l|c|c|}
\hline \multicolumn{1}{|c|}{ Parameter } & $\begin{array}{c}\text { Orthosis } \\
\text { part 1 }\end{array}$ & $\begin{array}{c}\text { Orthosis } \\
\text { part 2 }\end{array}$ \\
\hline Estimated time [h] & $10: 22$ & $9: 50$ \\
\hline Actual time [h] & $13: 20$ & $12: 45$ \\
\hline Time difference [h] & $2: 58$ & $2: 55$ \\
\hline $\begin{array}{l}\text { Estimated material use } \\
\text { (both materials) [g] }\end{array}$ & 88.02 & 80.89 \\
\hline Actual material use [g] & 83.00 & 77.00 \\
\hline Mass difference [g] & 5.02 & 3.89 \\
\hline Mass of product [g] & 79.23 & 73.72 \\
\hline $\begin{array}{l}\text { Mass of starting paths, } \\
\text { raft and supports [g] }\end{array}$ & 3.77 & 3.28 \\
\hline
\end{tabular}

the thumb [21] and the other fingers [4]. In the case of the wrist orthosis, the latter are not important from the point of view of the further design, because the digital model is cut at the height of the metacarpus. In the opinion of the authors, it would be reasonable to develop a universal tool to fix the upper limb of the user during scanning. The scanning process itself should also be as short as possible to minimize the possibility of changes in the body position. This problem may be particularly important when scanning children.

During the modeling of the orthosis in the CAD system, no significant difficulties were encountered. However, it should be noted that the modeling was performed by a person with many years of experience in the CAD systems, but without the previous experience in the design of orthoses. The time needed to manually shape the orthosis could be shorter, but the final solution should be, if possible, a complete automation of this activity.

In the manufacturing of both parts of the orthosis, similar discrepancies were found between the estimated time and material consumption and actual values (Table 2). Importantly, the intervention of the FDM machine operator, related to stopping the manufacturing process or changing the value of its parameters, was never required. The orthosis, despite the small area of contact with the raft, did not detach from it, at the same time their separation after removing from the working chamber of the FDM machine required only a small manually applied force and was not problematic.

During production, a slight oozing was noticed only for the TPU material. However, this took place only in a few initial layers, i.e. when creating the raft, so it did not affect the quality of the surface of the orthosis. In some areas, a very slight stringing of the TPU material is visible; for ABS, this effect was not observed at all. These errors can be eliminated by changing the values of parameters related to retraction.

The area around the thumb was the only place where, due to the geometry of the orthosis protruding beyond the outline of the previous layer, it was necessary to use support structures (Figure 3 ). They were created correctly, fulfilled their task and could be easily removed. Of course, a more beneficial situation would be to completely eliminate the need for supports. However, it would require changes in the structure of the orthosis near the thumb and the surface of its division.

The thickness of the TPU part is so small that with the material width used $(0.4 \mathrm{~mm})$, the contour filling is practically absent, and the contours are not always adjacent to each other along their entire length. In some places, weaker binding of contours can be detected, which does not interfere with the use. According to the authors, it would be reasonable to limit the number of contours (shells) in the TPU part to one, or use, for this material only, an extrusion die with a smaller diameter and use a smaller path width. In the opinion of the authors, this would increase the strength of binding of the paths in the TPU layer.

The holes for the catches fixing the orthosis halves had to be made with material bridging. Thus, it was possible to avoid using supports in their interior. Out of 16 holes, only one had incor-

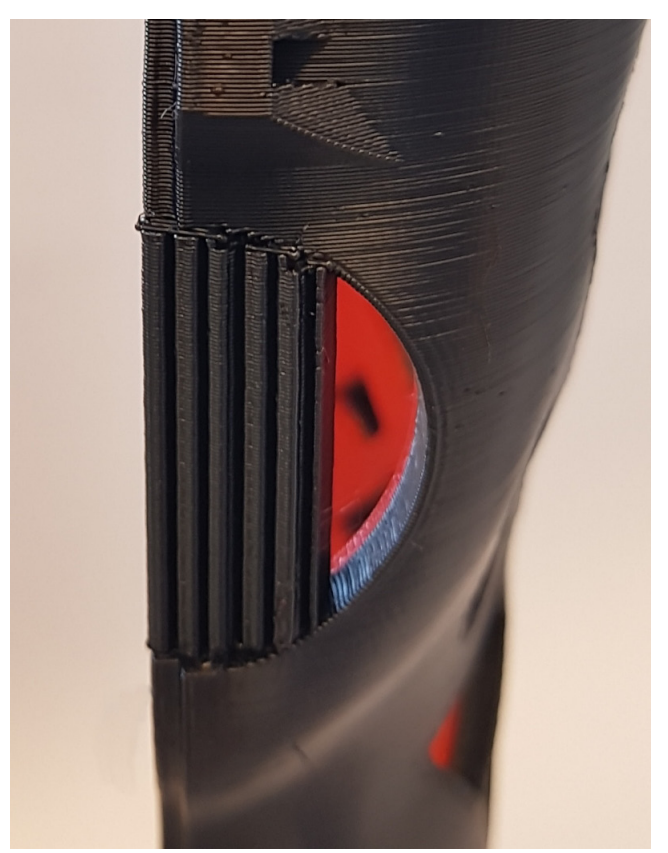

Fig. 3. Support in the thumb hole 
rectly deposed threads of material. However, they could be easily removed during postprocessing, without significant altering of the functionality of the hole. Each of the holes was loaded with a force of $100 \mathrm{~N}$. All the holes carried the load without signs of permanent deformation. It is by far more than the expected strength that can affect the orthosis in normal functioning.

The gouge at the edges of the splitting manufactured correctly and it significantly reduces the difficulty associated with self-installing the orthosis by the user, using only one hand. Moreover, the shape and dimensions of the gouging used form a tight connection between the orthosis halves. The force required to break this connection was measured as $22 \mathrm{~N}$ and thus relieves the holes for fixing hooks by that amount.

Both halves of the manufactured orthosis are shown in Figure 4. The total weight of the assembled orthosis was $152.95 \mathrm{~g}$. In the user's opinion, this is not a value that causes significant limb fatigue resulting from the necessity of lifting it. For comparison, a classic mechanical watch with a metal bracelet, worn in the same place, may weigh slightly less than the discussed orthosis. This supports the statement that in terms of weight, the orthosis is suitable for long-term use, without limiting the mobility of the limb as a whole.

The user assessed the fit of the created orthosis to the anatomy of his limb as correct. However, they pointed out that the area around the thumb was definitely the closest fit to the body, while the largest clearance occurred on the final section of the orthosis, near the elbow joint. The stiffness of the orthosis was assessed as high enough to properly block the wrist joint. Other mechanical properties of the orthosis allowed its proper use.

The user's suggestion was to apply a change in design of the area of the thumb, associated with pulling out the TPU layer, up to the outer surface

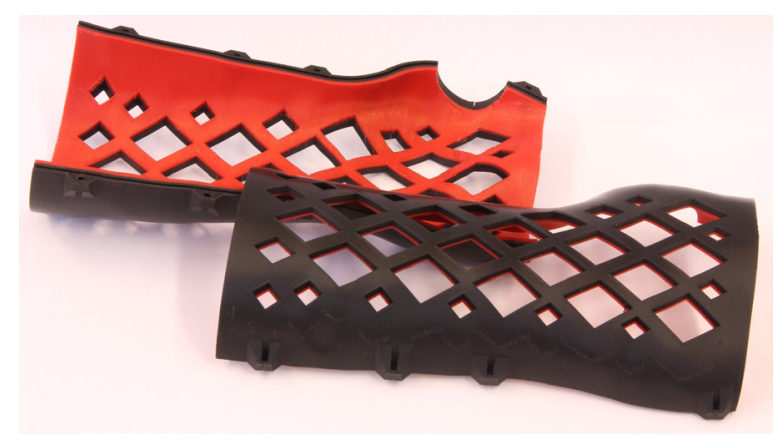

Fig. 4. Manufactured wrist orthosis
Table 3. The duration of individual stages

\begin{tabular}{|l|c|}
\hline \multicolumn{1}{|c|}{ Activity } & Time $[\mathrm{h}]$ \\
\hline Scanning & $0: 20$ \\
\hline Scan processing & $1: 30$ \\
\hline CAD modelling & $7: 00$ \\
\hline FDM manufacturing & $26: 05$ \\
\hline Post processing & $0: 10$ \\
\hline
\end{tabular}

of the product. This is the only place in the orthosis, where the skin of the user can easily have contact with the ABS material, which is definitely less comfortable than the TPU material.

The duration of individual work stages is shown in Table 3. The total time needed to complete all the activities leading to obtaining a physical model of a personalized orthosis was about 35 hours. It does not take into account the breaks that occurred between particular stages. It can be assumed that under the industrial conditions, with the same technical resources, it is possible to provide a personalized wrist orthosis to a patient within two working days.

Building a functionally personalized wrist orthosis with traditional methods of production would require from several (forming on the patient's body) hours to a dozen or so (forming on a positive casting) hours of work of an orthotics technician. Additive manufacturing is therefore significantly more time-consuming from the point of view of the occupation of machines. However, the load on the technician is already significantly lower at the present stage, and in the case of developing software for automatic orthosis design based on the data from patient scanning, the manual work load can be limited to a maximum of a dozen or so minutes per one product. Moreover, in the latter case, the employee will not be required to have high skills as orthotics technicians usually do, but only the ability to operate the scanner, the FDM machine and perform basic post processing of the additively manufactured elements. It should be noted that using the proposed method of manufacturing orthoses, the patient's participation in the production process will also be limited to only a few minutes, connected with the three-dimensional scan of the forearm.

An important social issue is the possibility of easy recycling of a personalized orthosis, which cannot be used by another patient. The strength of the ABS and TPU material combination in the orthosis provides the possibility of its proper use, but it can be a problem at the stage of product dis- 
posal. However, it should be noted, that the same problem applies to the orthoses made with traditional techniques, for which the production waste is definitely higher than in the FDM method. What is more, individual negative and positive forms also have to be recycled, thus increasing the ecological footprint.

\section{CONCLUSIONS}

The tests confirmed that it is possible to create a fully functional, multi-material wrist orthosis within one technological process. Owing to the design of the openwork and fixings, the material consumption, as well as the time needed for post processing operations have been significantly reduced. The combination of ABS and TPU materials proved to be strong enough to withstand the loads occurring during typical product use. At the same time, the use of elastic material on the inside of the orthosis has positively influenced the comfort of use. The gouging used on the split edge of both orthosis halves significantly facilitated the mutual positioning, and thus quick and easy insertion and removal of the orthosis by the user.

The problems indicated in the work should be a premise for further research. In addition to the need to thoroughly examine the mechanical properties of the combination of ABS and TPU, according to the authors, two research directions seem to be critical. The first one should lead to development of algorithms and, consecutively, computer software, which, based on a raw threedimensional scan of the patient's hand and accumulated medical knowledge, would be able to automatically model the digital geometry of the wrist orthosis. The second area of research should be focused on the FDM process, for which parameter values should be found that - while maintaining the minimum required functional properties of the orthosis - would minimize the time necessary for its production. The indicated directions of research are particularly important from the point of view of possible massive adoption of additive manufacturing in the production of individualized orthopedic supplies.

\section{Acknowledgements}

The studies were realized with a support from Polish National Center for Research and Devel- opment, in the scope of the "LIDER" program (grant agreement no. LIDER/14/0078/L-8/16/ NCBR/2017) and statutory activity financed by Polish Ministry of Science and Higher Education (02/23/DSPB/8716).

\section{REFERENCES}

1. Abilgaziyev A., et al., "Design and development of multi-nozzle extrusion system for 3D printer," 2015 International Conference on Informatics, Electronics \& Vision (ICIEV), Fukuoka, 2015, pp. 1-5, doi: 10.1109/ICIEV.2015.7333982.

2. Andringa A., et al., "Long-term use of a static hand-wrist orthosis in chronic stroke patients: a pilot study." Stroke research and treatment vol. 2013 (2013): 546093. doi:10.1155/2013/546093.

3. Banaszewski J., et al., 3D printed models in mandibular reconstruction with bony free flaps, Journal Of Materials Science-Materials In Medicine, 2018, vol. 29, issue 2, DOI: 10.1007/s10856-018-6029-5.

4. Baronio G., et al. A Critical Analysis of a Hand Orthosis Reverse Engineering and 3D Printing Process, Applied bionics and biomechanics vol. 2016 (2016): 8347478. doi:10.1155/2016/8347478.

5. Baronio G., Volonghi P., Signoroni A., Concept and Design of a 3D Printed Support to Assist Hand Scanning for the Realization of Customized Orthosis, Applied Bionics and Biomechanics, vol. 2017, Article ID 8171520, 8 pages, 2017. https://doi. org $/ 10.1155 / 2017 / 8171520$.

6. Belokar R.M., Banga H.K., Kumar R., A Novel Approach for Ankle Foot Orthosis Developed by Three Dimensional Technologies. In: IOP Conference Series: Materials Science and Engineering [Internet]; 2017, DOI: 10.1088/1757899X/280/1/012030.

7. Bijadi S, et al., Application of Multi-Material 3D Printing for Improved Functionality and Modularity of Open Source Low-Cost Prosthetics: A Case Study. ASME. Frontiers in Biomedical Devices, 2017 Design of Medical Devices Conference ():V001T10A003. doi:10.1115/DMD2017-3540.

8. Chimento J., Highsmith M.J., Crane N., 3D printed tooling for thermoforming of medical devices, Rapid Prototyping Journal, 2011, Vol. 17 Issue: 5, pp. 387-392, https://doi. org/10.1108/13552541111156513.

9. Davis F.J., Mitchell G.R. (2008) Polyurethane based materials with applications in medical devices. In: Bártolo, P. and Bidanda, B. (eds.) Bio-materials and prototyping applications in medicine. Springer, New York, pp. 27-48. ISBN 9780387476827. 
10. Espalin D., et al. "Multi-material, multi-technology FDM: exploring build process variations", Rapid Prototyping Journal, 2014, Vol. 20 Issue: 3, pp.236244, https://doi.org/10.1108/RPJ-12-2012-0112.

11. Faustini M.C. et al., Manufacture of Passive Dynamic ankle-foot orthoses using selective laser sintering, IEEE Trans Biomed Eng. 2008 Feb; 55 (2 Pt 1): 784-790. doi: 10.1109/TBME.2007.912638.

12. Górski F., et al., Selection of Fused Deposition Modeling Process Parameters using Finite Element Analysis and Genetic Algorithms, Journal Of Multiple-Valued Logic And Soft Computing, 2019, vol. 32, issue 3/4, pp. 293-311.

13. Guo R., et al. "Electrical and Thermal Conductivity of Polylactic Acid (PLA)-Based Biocomposites by Incorporation of Nano-Graphite Fabricated with Fused Deposition Modeling." Polymers, vol. 11, no. 3, 2019, doi:10.3390/polym11030549.

14. Haryńska, A., et al., Fabrication and Characterization of Flexible Medical-Grade TPU Filament for Fused Deposition Modeling 3DP Technology. Polymers 2018, 10, 1304.

15. Khondoker M.A.H., Sameoto D., Design and characterization of a bi-material co-extruder for Fused Deposition Modeling. In: ASME International Mechanical Engineering Congress and Exposition, Proceedings (IMECE), DOI: 10.1115/ IMECE201665330.

16. Kuipers T., Doubrovski E., Verlinden J., 3D hatching: linear halftoning for dual extrusion fused deposition modeling. In Proceedings of the 1st Annual ACM Symposium on Computational Fabrication (SCF '17). ACM, New York, NY, USA, 2017, Article 2, 7 pages. DOI: https://doi. org/10.1145/3083157.3083163.

17. Li, J. \& Tanaka, H., Feasibility study applying a parametric model as the design generator for 3D-printed orthosis for fracture immobilization, 3D Printing in Medicine (2018) 4: 1. https://doi. org/10.1186/s41205-017-0024-1.

18. Lopes L.R., Silva A.F., Carneiro O.S., Multimaterial 3D printing: The relevance of materials affinity on the boundary interface performance,
Additive Manufacturing, Volume 23, 2018, Pages 45-52, ISSN 2214-8604, https://doi.org/10.1016/j. addma.2018.06.027.

19. Mavroidis C., et al., Patient specific ankle-foot orthoses using rapid prototyping, Journal of NeuroEngineering and Rehabilitation, 2011, 8:1, https:// doi.org/10.1186/1743-0003-8-1.

20. Munguia J., Dalgarno K., Ankle foot orthotics optimization by means of composite reinforcement of free-form structures, 24th International SFF Symposium - An Additive Manufacturing Conference, SFF 2013, Pages 766-776.

21. Palousek D., et al., Pilot study of the wrist orthosis design process, Rapid Prototyping Journal, 2014, vol. 20, issue: 1, pp. 27-32, https://doi.org/10.1108/ RPJ-03-2012-0027.

22. Paterson, A., et al., Comparing additive manufacturing technologies for customised wrist splints. Rapid Prototyping Journal, 2015, 21 (3), pp. 230-243.

23. Ready S., Whiting G., Ng T. N., Multi-Material 3D Printing, NIP \& Digital Fabrication Conference, 2014 International Conference on Digital PrintingTechnologies, pp. 120-123(4).

24. Silva, M., et al., An alternative method to produce metal/plastic hybrid components for orthopedics applications. Proceedings of the Institution of Mechanical Engineers, Part L: Journal of Materials: Design and Applications, 2017, 231(1-2), 179186. https://doi.org/10.1177/1464420716664545.

25. Wierzbicka N, et al., Prototyping of Individual Ankle Orthosis Using Additive Manufacturing Technologies. Advances in Science and Technology Research Journal. 2017;11(3):283-288. doi:10.12913/22998624/76070.

26. Zawadzki P., Żywicki K., Smart product design and production control for effective mass customization in the Industry 4.0 concept, Management and Production Engineering Review, 2016, 7 (3), 105-112.

27. https://www.evilldesign.com/cortex

28. https://www.orfit.com/blog/the-volar-wrist-cockup-orthosis/

29. https://www.xkelet.com 